\title{
Design of the Polish Colonoscopy Screening Program: a randomized health services study
}

Authors

Institution
Michal F. Kaminski, Ewa Kraszewska, Maciej Rupinski, Milena Laskowska, Paulina Wieszczy, Jaroslaw Regula

Department of Gastroenterology, Hepatology and Oncology, Medical Centre for Postgraduate Education and the Maria Sklodowska-Curie Memorial Cancer Centre and Institute of Oncology, Warsaw, Poland submitted:

3. December 2014 accepted after revision: 20. June 2015

\section{Bibliography}

DOI http://dx.doi.org/

10.1055/s-0034-1392769

Published online: 30.10 .2015

Endoscopy 2015; 47: 1144-

1150

(c) Georg Thieme Verlag KG

Stuttgart · New York

ISSN 0013-726X

\section{Corresponding author}

\section{Michal F. Kaminski MD, PhD}

Department of

Gastroenterological Oncology Institute of Oncology

Roentgen Street 5

02-781 Warsaw

Poland

Fax: +48-22-5463035

mfkaminski@coi.waw.pl
Background and study aims: Colonoscopy screening for colorectal cancer has been implemented without evidence from randomized controlled trials quantifying its benefit and invariably as an opportunistic program, both of which are contrary to the European Union guideline recommendations. The aim of this paper is to describe the rationale and design of the first population-based colonoscopy screening program (PCSP), which was launched in Poland in 2012 as a randomized health services (RHS) study.

Methods: The PCSP is a natural extension of opportunistic colonoscopy screening implemented in 2000. It uses colonoscopy capacity, a quality assurance program, and a network of 92 centers built up during the opportunistic screening phase to develop a countrywide PCSP. Within the PCSP, single screening colonoscopy is offered to a target population aged 55-64 years. The PCSP uses an RHS design, which means that eligible individuals

\section{Introduction}

Colorectal cancer (CRC) is the second leading cause of cancer death in Poland and Europe $[1,2]$. Most CRCs develop from nonmalignant precursor lesions called adenomas over a long period of time. This slow development provides an opportunity for intervention with screening tests, which may result in decreased CRC incidence and mortality by removal of polyps or detection of CRC at an early stage. Although colonoscopy is approved by various expert committees as a primary screening tool for the prevention and early detection of CRC [3,4], no randomized trial has quantified the possible benefit of colonoscopy screening. Three such trials have been launched recently $[5,6]$ (NCT01239082), but their results will not be available within the next 10 years.

Once the benefit of cancer screening has been proven in a randomized trial, a challenge then exists to measure its true effectiveness following drawn from population registries are randomly assigned to immediate or postponed invitation to screening. Individuals from birth cohorts that will reach the upper age limit for screening before full implementation of the PCSP are randomly assigned, in a 1:1:1 ratio, to "immediate" screening, "postponed" screening, or a "never invited" control group. The RHS design is a natural platform that will evaluate the effectiveness of screening, and compare different age ranges for screening, invitation procedures, and quality improvement interventions. Up to 2015, 24 centers have been developed, with $34.2 \%$ geographic coverage and 851535 individuals enrolled.

Conclusions: The PCSP sets an example for implementation of population-based colonoscopy screening with experimental design to ensure proper evaluation of its effectiveness.

RHS registration number: 007_2015_1_RHS.

countrywide implementation [7]. Available comparison groups usually do not take into account chronologic changes in risk factors, cancer awareness, and advances in treatment [7]. It has therefore been suggested that CRC screening should be implemented using an experimental design in order to ensure the proper and detailed evaluation of its effects and feasibility as a public health policy $[8,9]$. This approach has been successfully introduced into the Finnish CRC screening program using fecal occult blood testing [10], and is currently being piloted in Norway in a sigmoidoscopy screening program [9].

The aim of this paper is to describe the rationale and design of the first population-based colonoscopy screening program (PCSP) for CRC, which was launched in Poland in 2012 as a randomized health services (RHS) study. 


\section{Program design}

\section{Building screening colonoscopy capacity}

The Polish PCSP is a natural extension of the opportunistic colonoscopy-based CRC screening program implemented in 2000 [11]. Within the framework of the opportunistic colonoscopybased program, a countrywide network of screening centers was gradually built up. In each screening center, an administrative office and a leading colonoscopist (usually the head of the endoscopy unit, responsible for coordination and supervision of the program locally) were established. Over the same period, the number of colonoscopists engaged in the program increased nearly 10 -fold. This was facilitated by establishing an initiative called the Polish School of Colonoscopy, which provided 2-4 weeks of hands-on training in colonoscopy for nearly 250 endoscopists. As a result of the training courses, the estimated overall colonoscopy capacity, as declared by the screening centers, reached 140000 procedures per annum in 2011, although the actual number of procedures performed was approximately 3 times lower because of funding limitations. This capacity was considered sufficient to cover once-in-a-lifetime screening colonoscopy with $30 \%$ compliance of the target population.

A screening program database was used to establish a colonoscopy quality assurance program from the start of the screening program. All colonoscopy records were reviewed annually by dedicated staff at the coordinating center in order to ensure complete follow-up of screen-detected lesions. The experiences of screening participants were evaluated immediately after screening colonoscopy using a dedicated questionnaire, but this information was not fed back to the screening centers. Since 2006, the quality assurance program also included feedback to the centers on colonoscopy quality indicators $[12,13]$.

In the decade since 2000, a solid basis in terms of staff, utilities, and procedures has been established through the opportunistic program, making a population-based program possible. In 2011, the Polish Ministry of Health agreed to gradually implement the population-based program using the previously developed opportunistic screening centers.

\section{Program coordination and funding}

A coordination unit is responsible for planning, executing, and evaluating the PCSP. The Polish Ministry of Health provides overall supervision of the program and covers the cost of program coordination and screening procedures (colonoscopies, histopathology, bowel preparation, and sedation if needed).

The screening centers are responsible for the local organization and performance of the screening colonoscopies. Each screening center provides the screening service to a target area of 2-10 surrounding poviats (administrative areas), which are assigned by the coordination unit based on colonoscopy capacity at the center. Target areas are within $40 \mathrm{~km}$ of the screening center. A gradual increase in the number of centers involved in the PCSP entails expansion of the programs' target areas, and full geographic coverage of the country is expected by 2021 (380 poviats with an estimated population of 5400000 individuals aged 55 64 years).

The PCSP is run in consecutive 1 - 2-year editions. Centers willing to participate in a new PCSP edition are selected by open bid. Preference is given to centers that participated in previous editions of the program and achieved a good recommendation from the coordination unit. Selected screening centers receive a contract covering all of the costs associated with the screening colonosco- pies for the assigned target population as well as access to a central web-based database system (SI-PBP), which handles screening invitations, colonoscopy appointments, colonoscopy and histopathology reports, and follow-up data.

\section{Target population and screening policy}

All individuals aged 55-64 years living in the target area for each screening center are eligible for the program. The only exclusion criteria are diagnosis of CRC registered in the National Cancer Registry or screening colonoscopy performed within the previous 10 years, as verified from the opportunistic colonoscopy screening program database.

The screening method offered to the target population is once-ina-lifetime colonoscopy. This decision is based on the concept that a high-quality colonoscopy can be used as a triage test at the age of about 60 years [14]. Individuals with precancerous lesions are recommended to undergo postpolypectomy surveillance according to the adapted European guidelines [15]; however, organization and financing of surveillance are based on the general healthcare system and are separate from the screening program. Individuals with a negative colonoscopy do not require further screening or surveillance. The concept of not repeating colonoscopy following a negative procedure relies on its long protective effect, which certainly exceeds 12 years [16] and probably 20 years [17], and an estimated life expectancy of 20 years for 60year-old individuals in Poland.

Each year, the coordination unit acquires from the population registry (PESEL Registry) a list of eligible individuals in the PCSP target areas, and mails a letter of invitation with a scheduled appointment date for colonoscopy to every individual randomized to the "immediate screening group" (see Implementation as an RHS study, below). Letters of invitation accompanied with an information folder and reply form are mailed 6-7 weeks prior to the scheduled appointment. Respective screening centers mail a reminder letter to nonresponders 3 weeks prior to the scheduled appointment. The screening center manages the invitees' responses, confirms appointment dates, and issues bowel preparation to participants who accept the invitation. The invitation scheme follows the framework developed within the NordICC study [5]. The offer of screening colonoscopy remains open for invitees until the age of 64 years. Currently, no re-invitations or alternative screening options are considered for nonresponders.

A screening colonoscopy and a split-dose 4-L polyethylene glycol (PEG) bowel preparation are offered free of charge to all invitees. The use of sedation is based on local screening center policy, but it has to be provided free of charge up to $20 \%$ of invitees upon their request. At colonoscopy, all detected precursor lesions are removed immediately whenever feasible, and other pathological findings are biopsied. The decision to remove more difficult lesions ( $>1 \mathrm{~cm}$ in size) immediately or to remove them during a dedicated therapeutic procedure is left to the discretion of the endoscopist. Insertion of the colonoscope is discontinued if an individual expresses significant discomfort or a wish to stop.

All histopathology results are entered into the SI-PBP database by collaborating histopathologists. The endoscopist performing the screening colonoscopy is responsible for categorizing the most advanced findings [11], issuing the surveillance recommendations [4], obtaining follow-up data from subsequent treatment, and entering all of the information into the SI-PBP database. 


\section{Implementation as an RHS study}

The PCSP has been implemented as an RHS study [18], with randomization at the individual level and a gradually expanding invitation scheme ( $\bullet$ Fig. 1 ). Each year $10 \%$ of each eligible birth cohort is randomly assigned to the "immediate screening group" and $10 \%$ to the "postponed screening group" (screening delayed for 5 years). The postponed screening group is not informed about their status. Randomization is stratified by birth cohort, sex, and poviat, with personal 1:1 matching within the strata. The invitation scheme assures that, once fully implemented, screening is offered to all eligible individuals from the target areas in 10 consecutive annual batches.

During the implementation phase, however, the screening may not be offered to all individuals from the 56-64-year-old birth cohorts, because some individuals will reach the age limit for screening before having a chance to be invited. Therefore, it was decided to construct, with no ethical reservations, a "never invited control" group by randomly drawing in advance one batch of individuals aged 56-64 years who will not be offered screening because of age restrictions. Therefore, at the first annual edition of the PCSP, $3 / 10$ of the birth cohort aged 56-64 years has been individually randomized in a 1:1:1 ratio to "immediate" screening, "postponed" screening (delayed for 5 years) or "never invited" control group. The birth cohort of 55 years has been individually randomized in a 1:1 ratio to either immediate screening or postponed screening (delayed for 5 years) ( $\bullet$ Fig. 2). This method ensures that starting from the 55-year-old cohort at the first edition, entire consecutive birth cohorts will be offered screening within 10 years. The details of the invitation scheme are presented in 0 Fig. 1.

\section{Quality assurance}

All screening centers entering the PCSP are selected through a competitive bidding process. Eligible endoscopy centers have to fulfil the following inclusion criteria: 1) participation in a previous edition of the PCSP or the opportunistic colonoscopy screening program; 2) declared ability to perform at least 1000 screening colonoscopies in addition to routine clinical colonoscopies; 3 ) minimum quality assurance criteria confirmed by the program coordinator (adenoma detection rate of $\geq 20 \%$, cecal intubation rate of $\geq 95 \%$, rate of high grade dysplasia in adenomas $<10 \mathrm{~mm}$ of $\leq 1 \%$ ); and 4 ) participating endoscopists with gastroenterology specialty or Certificate of Competence in Colonoscopy issued by the Polish Society of Gastroenterology.

At the end of each edition of the PCSP, the SI-PBP database is reviewed to assess the completeness of data, the experience of screenees as reported on the Gastronet questionnaire [19], and to calculate quality indicators for centers and individual endoscopists. Following the review, feedback information on performance is sent to each screening center. In addition, all endoscopists who express their interest in getting individual performance data receive email feedback on colonoscopy quality indicators presented in a league table, which enables comparison with anonymous results of all endoscopists who performed at least 30 colonoscopies.

Screening center leaders are invited to participate in a 2-day hands-on train-the-leaders course [20]. The course is aimed at improving their center quality and the quality of their colonoscopy training skills. Histopathologists who overdiagnose high grade dysplasia in small and diminutive polyps (>1\%) are invited for a re-training session with expert gastrointestinal pathologists at the coordinating center.

\section{Ethical issues}

This is an RHS study, which is similar in design to a randomized controlled trial (RCT) but has several differences especially in terms of ethical issues. No formal bioethical committee approval is required but the PCSP is regulated and overseen by the health authority (the Polish Ministry of Health), which approves both screening colonoscopy (when possible) and no screening (not realistic to provide for all citizens at this moment in time). This regulation fulfils the ethical requirement of RHS studies [18]. All co-studies (see below) are either approved by the central Bioethical Committee at the Center of Oncology Institute and Medical Centre for Postgraduate Education, or have been granted exemption from this approval.

Individuals are randomly assigned to the immediate screening group, postponed screening group, or the never invited control group, without written informed consent. All individuals who are invited for screening, however, must provide written informed consent before they undergo colonoscopy.

There are several reasons why this approach was considered ethical. Screening colonoscopy cannot be offered to the entire target population at the same point in time. The most ethical way of selecting the order of invitation is by random draw. Therefore, each year, $10 \%$ of the target population is randomly selected to the immediate screening group to ensure that in 10 years' time $100 \%$ of the target population has been offered screening. During the implementation phase, however, some individuals will reach the upper age limit for screening before having a chance to be invited. As colonoscopy screening has never been studied in a randomized controlled trial, it has been decided to randomly select a never invited control group from the birth cohorts that will reach the upper age limit for screening in order to fully investigate the effectiveness of colonoscopy in the population during implementation. Furthermore, with an ongoing development of medical treatment, screening colonoscopy may shift from being effective to being ineffective, and there is no way of proving this other than by using an RHS design.

\section{Program evaluation}

$\nabla$

\section{Long term outcomes}

The primary outcome measures of the PCSP are CRC incidence and incidence-based CRC mortality. The RHS design allows the following analyses to be performed.

1. Cumulative CRC incidence and mortality during 10 years $(15,20,25$ years) of follow-up in individuals aged $56-64$ years who are randomized at the first edition of the implementation phase to immediate screening or the never invited control group.

2. Cumulative $\mathrm{CRC}$ incidence and mortality during 10 years $(15,20,25$ years) of follow-up in individuals aged $60-64$ years who are randomized at editions $1-5$ of the implementation phase to immediate screening or control (defined as those randomized to the never invited group plus those randomized to postponed screening who reached the age limit before they could be invited).

3. Cumulative CRC incidence and mortality during 10 years $(15,20,25$ years) of follow-up in individuals invited for screening in the implementation phase at the age of $55-59$ years vs. $60-64$ years.

Data on CRC incidence, CRC mortality, and overall mortality will be obtained by matching individuals against the National Cancer 


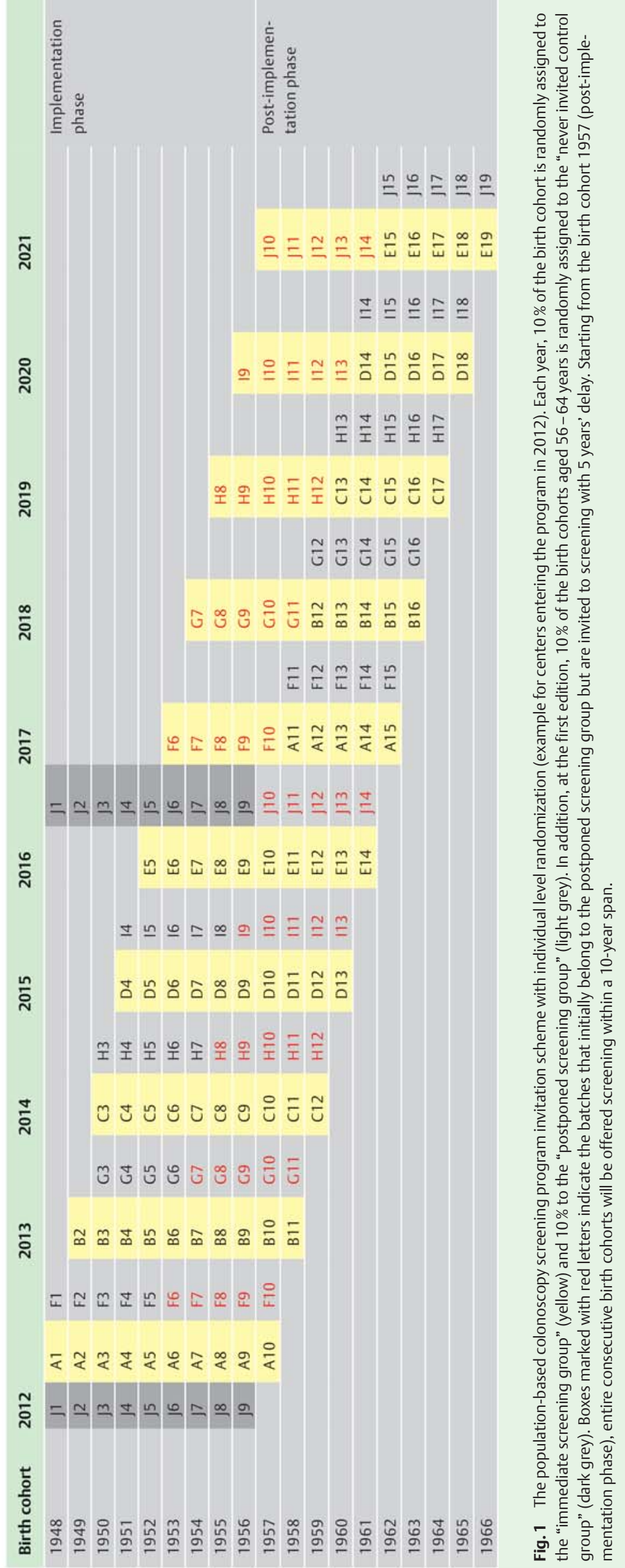




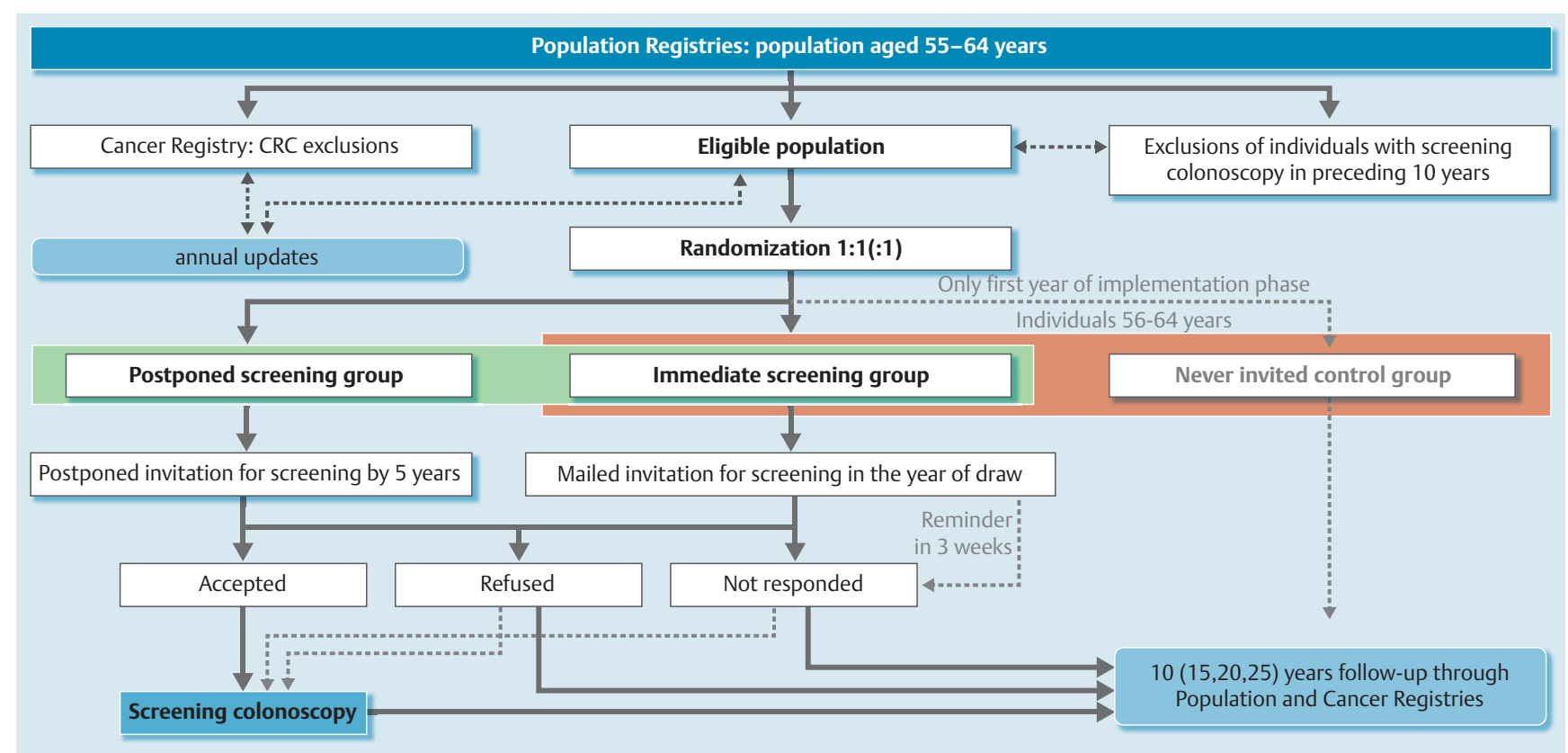

Fig. 2 The Polish Colonoscopy Screening Platform: recruitment of participants. The pink-shaded box indicates individuals aged 56-64 years who will be randomized at the first edition of the implementation phase to the screening (immediate screening) or control (never invited control) groups and compared in terms of cumulative colorectal cancer (CRC) incidence and mortality during the follow-up time. The green-shaded box indicates individuals aged $55-64$ years who will be randomized to the immediate screening or postponed screening groups and compared in terms of cumulative CRC incidence and mortality (and surrogate end points) during the follow-up time. Dotted arrows indicate that the offer of screening colonoscopy remains open for invitees until the age of 64 years, even if they initially refuse or do not respond.

Registry database using unique personal PESEL number. Records on death status in the National Cancer Registry database are updated by personal matching against the PESEL Registry. The CRC incidence and mortality rates will be compared between randomized groups using the intention-to-screen principle.

Moreover, as a result of gradual geographic expansion of the PCSP over 10 years, it will be possible to create parallel groups in poviats with an established PCSP, poviats with only an opportunistic colonoscopy screening program, and those with no program at all. The use of these parallel groups and their historical controls (similar to the situation described in mammography screening in Norway [7]) will enable comparison between populationbased and opportunistic colonoscopy screening programs.

\section{Short term outcomes}

The PCSP is accompanied by several co-studies, which constitute the study "Experimental population-based platform to evaluate and monitor the effectiveness of screening colonoscopy: a population-based comparative effectiveness study" (for a more detailed description see Appendix e1, available online). The costudies are designed to evaluate the implementation phase of the program.

1. The sensitivity of the screening episode and screening program for detection of CRC expected to become clinically detectable within 2 years (or 5 years).

2. The incidence of advanced CRC in the screening and control groups within 2 years (or 5 years) from the date of draw.

3. A 30-day mortality rate and 30-day hospitalization rate in the screening and control groups.

4. The cost-effectiveness of the PCSP.

5. Participation in screening colonoscopy in response to various invitation procedures. In particular, an RCT will be conducted to evaluate the effect of the advanced notification letter (ANL) and low-volume bowel preparation compared with standard invitation and standard split-dose 4-L PEG bowel preparation, respectively.

6. The effect of routine video recording of screening colonoscopy on quality indicators.

\section{Sample size}

This is an RHS study, which means that the recruitment process will be permanently ongoing unless the health policy is changed, and thus the sample size is virtually unlimited. Nevertheless, a sample size was calculated for the number needed to show the difference in the study primary outcome measures. In Poland, the expected accumulated CRC incidence-based mortality at 15 years' follow-up in the nonscreened population aged 55-64 years is $1.02 \%$ (1021 deaths per 100000 person-years). In the intention-to-screen analysis, a 10\% reduction in CRC mortality (assuming, conservatively, $50 \%$ efficacy and $20 \%$ attendance rate) at 15 years' follow-up was considered to be clinically important and possible to achieve. In order to assure $80 \%$ power to detect this difference at a one-sided 5\% significance level, 144644 individuals are needed for screening and the same number are to be identified as controls.

\section{Current status and future perspectives}

$\nabla$

The PCSP was launched in July 2012 in five centers, covering the target screening area of 12 poviats (3.2\% of the total 380 poviats in Poland). The PCSP was further expanded to 10 centers (34 poviats, $8.9 \%$ ) in 2013, 20 centers (68 poviats, 17.9\%) in 2014, and will reach 24 centers ( 130 poviats, $34.2 \%$ ) in 2015.0 Fig. 3 shows the gradual geographic expansion of the PCSP in Poland. Between 2012 and 2015, a total of 346319 individuals have been random- 


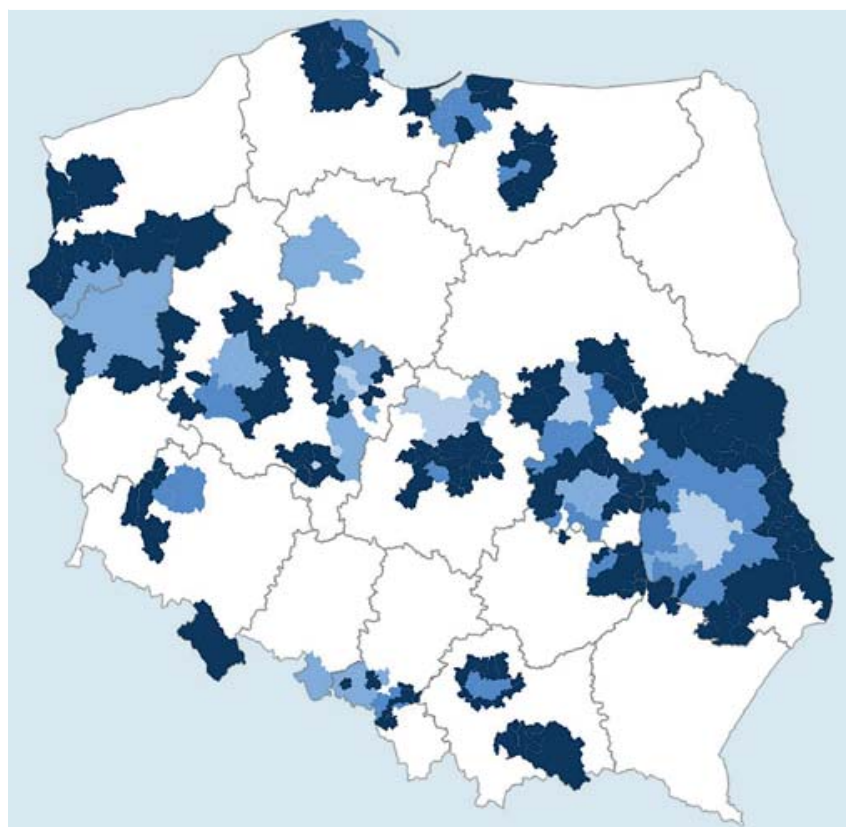

Fig. 3 Geographic expansion of the population-based colonoscopy screening program in Poland. Lightest blue areas represent poviats starting in 2012, light blue in 2013, medium blue in 2014, and dark blue in 2015 .

ly assigned to the immediate screening group, 346242 to the postponed screening group, and 158974 to the never invited control group.

The first 5 years of the PCSP implementation are expected to enrol 35 screening centers, achieve $40 \%$ geographic (poviat) coverage, and invite a total of 500000 individuals to screening colonoscopy. Further gradual increase in the number of screening centers is planned up to about 130 , with full geographic coverage by 2021 .

\section{Discussion}

\section{$\nabla$}

This paper describes the design of the first population based colonoscopy screening programme implemented as randomized health services study.

Colonoscopy screening has invariably been implemented as an opportunistic or nonorganized program [21], which is not in accordance with European Union guideline recommendations [4]. The PCSP design shows how to take advantage of previously implemented opportunistic screening to build up sufficient, high-quality colonoscopy capacity for population-based screening. It also proposes how to transform a network of developed opportunistic screening centers into a population-based program with gradually increasing geographic coverage. In order to fit the invitation scheme with colonoscopy capacity, the geographic expansion of the population-based program might be accompanied by gradual enrollment of consecutive fractions of each birth cohort. This solution allows full benefit from the fact that screening colonoscopy does not need to be repeated frequently or even at all. Random draw is probably the most ethical method of selecting the order of birth cohort fractions to be invited for screening and gives an opportunity for proper evaluation of screening effects using experimental design [8,9].

The RHS design of the PCSP is expected to provide unbiased evaluation of program effectiveness. It is particularly relevant for co- lonoscopy screening, which is invasive, inconvenient, and costly, as this method of screening has not been appropriately tested in a randomized trial [5]. Moreover, proper measurement of screening effectiveness following implementation as a nationwide health policy is challenging without experimental design because of dynamic changes in background CRC epidemiology [22]. This type of challenge was encountered in the United States, where the actual decline in CRC incidence and mortality preceded the expected time of decline following implementation of colonoscopy screening [23]. The PCSP design allows the study of surrogate end points that could reliably predict CRC mortality reduction sooner and therefore could be used to refine a health policy many years in advance [24]. Finally, the design of the PCSP provides a natural platform for short-term studies on fine tuning the screening policy.

The PCSP design has some limitations, however. First, its implementation is a long-lasting process that requires extensive database management support and sustained participation of enrolled screening centers. Second, the 5-year difference in invitation to screening time between immediate screening and postponed screening groups may not be long enough to investigate surrogate end points of screening efficacy such as incidence of advanced CRC. Third, study end points will be derived from cancer registries, which are known to be incomplete, although end points will be ascertained using the same imperfect tool and so the effect estimates of the screening intervention should be valid. In summary, this paper describes the rationale and design of the first PCSP for CRC implemented as an RHS study. This may set an example for the growth of colonoscopy capacity, introduction of quality assurance, gradual expansion of population coverage, and proper evaluation of the effectiveness of organized colonoscopy screening programs.

Competing interests: Dr. Kaminski has received teaching and speaking fees from Olympus Polska.

\section{Acknowledgments}

$\nabla$

The Program is supported by the Polish Ministry of Health (costs of screening procedures, screening administration and database management [partially]) and by a grant (database management and scientific evaluation of the program) from the Foundation of Polish Science (TEAM/2012-9/5) financed by EU structural funds, Innovative Economy Operational Programme 2007-2013. Michal F. Kaminski received a stipend from the Polish Ministry of Science and Higher Education ( $77 / 2012)$ during the study period (2012-2015).

The authors thank Tomasz Matwiejczuk and Marcin Zdunik, from the IQ5 Ltd., Bialystok, Poland, for their outstanding database management.

\section{References}

1 Ferlay J, Parkin DM, Steliarova-Foucher E. Estimates of cancer incidence and mortality in Europe in 2008. Eur J Cancer 2010; 46: 765 - 781

2 Wojciechowska U, Didkowska J, Zatoński W. Nowotwory złośliwe w Polsce w 2008 roku. Cancer in Poland in 2008. Gdańsk: Centrum Onkologii-Instytut; 2010

3 Force USPST. Screening for colorectal cancer: U.S. Preventive Services Task Force recommendation statement. Ann Intern Med 2008; 149: 627-637

4 Segnan N, Patnick J, von Karsa L. European guidelines for quality assurance in colorectal cancer screening and diagnosis. Luxembourg: Publications Office of the European Union; 2010 
5 Kaminski MF, Bretthauer M, Zauber AG et al. The NordICC Study: rationale and design of a randomized trial on colonoscopy screening for colorectal cancer. Endoscopy 2012; 44: 695-702

6 Quintero E, Castells A, Bujanda L et al. Colonoscopy versus fecal immunochemical testing in colorectal-cancer screening. N Engl J Med 2012; 366: $697-706$

7 Kalager $M$, Zelen $M$, Langmark $F$ et al. Effect of screening mammography on breast-cancer mortality in Norway. N Engl J Med 2010; 363: $1203-1210$

8 Malila N, Anttila A, Hakama M. Colorectal cancer screening in Finland: details of the national screening programme implemented in Autumn 2004. J Med Screen 2005; 12: 28-32

9 Bretthauer M, Hoff G. Comparative effectiveness research in cancer screening programmes. BMJ 2012; 344: e2864

10 Malila N, Oivanen T, Malminiemi $O$ et al. Test, episode, and programme sensitivities of screening for colorectal cancer as a public health policy in Finland: experimental design. BMJ 2008; 337: a2261

11 Regula J, Rupinski M, Kraszewska E et al. Colonoscopy in colorectal-cancer screening for detection of advanced neoplasia. N Engl J Med 2006; 355: $1863-1872$

12 Kaminski MF, Kraszewska E, Polkowski M et al. Continous quality improvement of screening colonoscopy: data from a large colorectal cancer screening program. Gastrointest Endosc 2009; 69: AB215

13 Kaminski MF, Regula J, Kraszewska $E$ et al. Quality indicators for colonoscopy and the risk of interval cancer. N Engl J Med 2010; 362: $1795-1803$

14 Bretthauer $M$, Kalager $M$. Colonoscopy as a triage screening test. N Engl J Med 2012; 366: 759-760
15 Kaminski MF, Regula J, Bartnik W et al. Polish Society of Gastroenterology guidelines for colonoscopy surveillance following polypectomy adapted European guidelines. Gastroenterologia Klin 2011; 2: 55-62

16 Schoen RE, Pinsky PF, Weissfeld JL et al. Colorectal-cancer incidence and mortality with screening flexible sigmoidoscopy. N Engl J Med 2012; 366: $2345-2357$

17 Brenner H, Chang-Claude J, Seiler CM et al. Long-term risk of colorectal cancer after negative colonoscopy. J Clin Oncol 2011; 29: 3761 - 3767

18 Hakama M, Malila N, Dillner J. Randomised health services studies. Int J Cancer 2012; 131: 2898-2902

19 Hoff G, Bretthauer M, Huppertz-Hauss G et al. The Norwegian Gastronet project: continuous quality improvement of colonoscopy in 14 Norwegian centres. Scand J Gastroenterol 2006; 41: 481 -487

20 Kaminski MF, Anderson J, Valori $R$ et al. Leadership in training to improve adenoma detection rate in screening colonoscopy: a nationwide randomized trial. Gut 2015: DOI 10.1136/gutjnl-2014-307503

21 Pox C, Schmiegel W, Classen M. Current status of screening colonoscopy in Europe and in the United States. Endoscopy 2007; 39: 168-173

22 Didkowska J, Wojciechowska U, Zatonski W. Prediction of cancer incidence and mortality in Poland up to the year 2025. Warsaw, Poland: The National Cancer Registry; 2009: Available from: http://onkologia. org.pl/publikacje/

23 Edwards BK, Ward E, Kohler BA et al. Annual report to the nation on the status of cancer, 1975-2006, featuring colorectal cancer trends and impact of interventions (risk factors, screening, and treatment) to reduce future rates. Cancer 2010; 116: 544-573

24 Cuzick J, Cafferty FH, Edwards $R$ et al. Surrogate endpoints for cancer screening trials: general principles and an illustration using the UK Flexible Sigmoidoscopy Screening Trial. J Med Screen 2007; 14: 178 185

\section{Supplementary Appendix e1}

online content viewable at:

http://dx.doi.org/10.1055/s-0034-1392769 